\title{
Improved three-dimensional color-gradient lattice Boltzmann model for immiscible two-phase flows
}

\author{
Z. X. Wen, Q. Li, ${ }^{*}$ and Y. Yu \\ School of Energy Science and Engineering, Central South University, Changsha 410083, China \\ Kai H. Luo \\ Department of Mechanical Engineering, University College London, Torrington Place, London WC1E 7JE, United Kingdom
}

(Received 29 March 2019; published 7 August 2019)

\begin{abstract}
In this paper, an improved three-dimensional color-gradient lattice Boltzmann (LB) model is proposed for simulating immiscible two-phase flows. Compared with the previous three-dimensional color-gradient LB models, which suffer from the lack of Galilean invariance and considerable numerical errors in many cases owing to the error terms in the recovered macroscopic equations, the present model eliminates the error terms and therefore improves the numerical accuracy and enhances the Galilean invariance. To validate the proposed model, numerical simulations are performed. First, the test of a moving droplet in a uniform flow field is employed to verify the Galilean invariance of the improved model. Subsequently, numerical simulations are carried out for the layered two-phase flow and three-dimensional Rayleigh-Taylor instability. It is shown that, using the improved model, the numerical accuracy can be significantly improved in comparison with the color-gradient LB model without the improvements. Finally, the capability of the improved color-gradient LB model for simulating dynamic two-phase flows at a relatively large density ratio is demonstrated via the simulation of droplet impact on a solid surface.
\end{abstract}

DOI: 10.1103/PhysRevE.100.023301

\section{INTRODUCTION}

In the past three decades, the lattice Boltzmann (LB) method [1-11], which originates from the lattice gas automaton (LGA) method [12], has been developed into an efficient numerical approach for simulating fluid flow and heat transfer. Different from conventional numerical methods, which are based on the direct discretization of macroscopic governing equations, the LB method is built on the mesoscopic kinetic equation. It tracks the evolution of a particle distribution function and then accumulates the particle distribution function to obtain the macroscopic properties. Owing to its kinetic nature, the LB method exhibits some advantages over conventional numerical methods. For example, in the LB equation the convective operator (the streaming process) is linear, whereas the convective terms of the Navier-Stokes equations are nonlinear [13]. Moreover, in the LB simulations the complex boundary conditions can be formulated with the elementary mechanical rules such as the bounce-back rule according to the interaction of the "LB particles" with the solid walls. Furthermore, the LB method is ideal for parallel computing because of its explicit scheme and the local interactions.

Since the emergence of the LB method, its applications to multiphase flows have always been a very important theme

\footnotetext{
*Corresponding author: qingli@csu.edu.cn
}

Published by the American Physical Society under the terms of the Creative Commons Attribution 4.0 International license. Further distribution of this work must maintain attribution to the author(s) and the published article's title, journal citation, and DOI. of this method and various multiphase LB models have been developed from different points of view [14]. Generally, most of the existing multiphase LB models can be classified into the following four categories [5-7], i.e., the color-gradient LB method, the pseudopotential LB method, the free-energy LB method, and the phase-field LB method. The first colorgradient LB model was proposed by Gunstensen et al. [15], who extended the multicomponent lattice gas model of Rothman and Keller [16] to the LB method [17]. In the colorgradient LB method, two distribution functions are introduced to represent two different fluids and a color-gradient-based perturbation operator is employed to generate the surface tension as well as a recoloring step for separating different phases or components. The pseudopotential LB method, which is the simplest multiphase LB method, was introduced by Shan and Chen [18,19]. In this method, the fluid interactions are mimicked by an interparticle potential, through which the separation of different phases or components can be achieved naturally. The free-energy LB method was developed by Swift et al. [20,21] based on thermodynamics considerations. They proposed to modify the second-order moment of the particle equilibrium distribution function so as to include a nonideal thermodynamic pressure tensor. The phase-field LB method is based on the phase-field theory, in which the interface dynamics is described by an order parameter that obeys the Cahn-Hilliard equation or a Cahn-Hilliard-like equation [22].

Each of these multiphase LB methods has its advantages and limitations. A comprehensive review of the pseudopotential LB method and the phase-field LB method can be found in Ref. [7]. In addition, the book by Huang et al. [14] is also dedicated to the multiphase LB methods. In this work, we restrict our study to the color-gradient two-phase LB method, 
which exhibits very low dissolution for tiny droplets or bubbles [23] in comparison with other two-phase LB methods. In the original color-gradient LB model devised by Gunstensen et al. [15], the work done by the color gradient against the color flux was maximized to force the colored particles to move toward fluids with the same color. In addition, the model of Gunstensen et al. suffers from the limitation of equal densities for two-phase flows. Some improvements have been conducted to overcome the shortcomings of the original colorgradient model. Grunau et al. [24] modified the form of the particle equilibrium distribution function to allow for variable density and viscosity ratios. Latva-Kokko and Rothman [25] replaced the numerical maximization recoloring step of the model of Gunstensen et al. with a formulaic segregation algorithm, which solves the lattice pinning problem at the interface region and significantly improves the computational efficiency of the color-gradient LB method.

Later, Reis and Phillips [26] proposed a new perturbation operator for generating the surface tension of the colorgradient LB method and derived a theoretical expression for the surface tension through its mechanical definition. Liu et al. [27] extended the model of Reis and Philips to threedimensional space by deriving a generalized perturbation operator, in which an expression for the surface tension parameter is directly obtained without approximations. However, similar to the free-energy two-phase LB method, the colorgradient two-phase LB method also modifies the equilibrium distribution function [26,27] to incorporate the pressure of fluid. Hence it also suffers from the lack of Galilean invariance [7]. Through the Chapman-Enskog analysis, Huang et al. [28] showed that some error terms exist in the macroscopic momentum equation recovered from the color-gradient twophase LB method. They demonstrated that for two-phase flows with different densities the error terms significantly affect the numerical accuracy. A scheme has been proposed by Huang et al. [28] to eliminate the error terms, but they emphasized that their scheme just works well for cases of density ratios less than 10. Recently, Ba et al. [29] developed a twodimensional multiple-relaxation-time (MRT) color-gradient LB model for two-phase flows. To eliminate the error terms in the macroscopic momentum equation, an extension of the approach of $\mathrm{Li}$ et al. [30] was made, which was devised for recovering $p=\rho R T$ in a double-distribution-function LB model on standard lattices for thermal compressible flows.

In the present work, we aim at proposing an improved three-dimensional color-gradient LB model for two-phase flows. The error terms in the momentum equation are removed following the approach of $\mathrm{Li}$ et al. [30]. To be specific, a high-order term is added to the equilibrium distribution function, through which the off-diagonal elements of the third-order moment of the equilibrium distribution function satisfy the required relationship for recovering the NavierStokes equations. Meanwhile, the deviations of the diagonal elements are corrected through introducing a correction term into the LB equation. The rest of the present paper is organized as follows. In Sec. II, the existing three-dimensional colorgradient LB models are briefly introduced. The improved three-dimensional (3D) color-gradient LB model is proposed in Sec. III. Numerical simulations are performed in Sec. IV to validate the improved model. Finally, Sec. V concludes the present paper.

\section{EXISTING 3D COLOR-GRADIENT LB MODELS}

\section{A. Color-gradient LB equation}

In the color-gradient LB method, the two immiscible fluids are represented by a red fluid and a blue fluid, respectively. The corresponding distribution functions are denoted by $f_{i}^{k}$, where $i$ is the lattice velocity direction and $k=R$ or $B$ denotes the color ("red" or "blue"). The total distribution function is defined as $f_{i}=f_{i}^{R}+f_{i}^{B}$. The evolution of the distribution functions is governed by the following LB equation [31]:

$$
f_{i}^{k}\left(\mathbf{x}+\mathbf{e}_{i} \delta_{t}, t+\delta_{t}\right)-f_{i}^{k}(\mathbf{x}, t)=\Omega_{i}^{k}(\mathbf{x}, t),
$$

where $\mathbf{x}$ is the spatial position, $\mathbf{e}_{i}$ is the discrete velocity in the $i$ th direction, $t$ is the time, $\delta_{t}$ is the time step, and $\Omega_{i}^{k}$ is the collision operator [26,31],

$$
\Omega_{i}^{k}=\left(\Omega_{i}^{k}\right)^{(3)}\left[\left(\Omega_{i}^{k}\right)^{(1)}+\left(\Omega_{i}^{k}\right)^{(2)}\right],
$$

where $\left(\Omega_{i}^{k}\right)^{(1)}$ is the single-phase collision operator, $\left(\Omega_{i}^{k}\right)^{(2)}$ is the perturbation operator, which is used to generate the surface tension, and $\left(\Omega_{i}^{k}\right)^{(3)}$ is the recoloring operator responsible for phase segregation and maintaining the phase interface [27,29]. When the Bhatnagar-Gross-Krook (BGK) collision operator is applied, the single-phase collision operator is given by

$$
\left(\Omega_{i}^{k}\right)^{(1)}=-\frac{1}{\tau}\left[f_{i}^{k}(\mathbf{x}, t)-f_{i}^{k, \mathrm{eq}}(\mathbf{x}, t)\right],
$$

where $\tau$ is the nondimensional relaxation time and $f_{i}^{k \text {, eq }}$ is the equilibrium distribution function of $f_{i}^{k}$. The macroscopic variables are calculated by

$$
\begin{aligned}
\rho_{R} & =\sum_{i} f_{i}^{R}, \quad \rho_{B}=\sum_{i} f_{i}^{B}, \quad \rho=\sum_{k} \rho_{k}, \\
\rho \mathbf{u} & =\sum_{i} \sum_{k} \mathbf{e}_{i} f_{i}^{k},
\end{aligned}
$$

where $\rho_{k}$ is the density of fluid $k, \rho$ is the total density, and $\mathbf{u}$ is the macroscopic velocity.

\section{B. 3D color-gradient LB models}

The first three-dimensional color-gradient LB model is attributed to Tölke et al. [31], who constructed a threedimensional 19-velocity (D3Q19) color-gradient LB model for immiscible two-phase flows based on the studies of Gunstensen et al. [15] and Grunau et al. [24]. The lattice velocities $\left\{\mathbf{e}_{i}\right\}$ of the D3Q19 lattice are given by

$$
\mathbf{e}_{i}=c\left[\begin{array}{rrrrrrrrrrrrrrrrrrr}
0 & 1 & -1 & 0 & 0 & 0 & 0 & 1 & -1 & 1 & -1 & 1 & -1 & 1 & -1 & 0 & 0 & 0 & 0 \\
0 & 0 & 0 & 1 & -1 & 0 & 0 & 1 & -1 & -1 & 1 & 0 & 0 & 0 & 0 & 1 & -1 & 1 & -1 \\
0 & 0 & 0 & 0 & 0 & 1 & -1 & 0 & 0 & 0 & 0 & 1 & -1 & -1 & 1 & 1 & -1 & -1 & 1
\end{array}\right]
$$


where $c=1$ is the lattice constant. The equilibrium distribution function is chosen as [31]

$$
f_{i}^{k, \text { eq }}=\rho_{k}\left\{\phi_{i}^{k}+\omega_{i}\left[\frac{3}{c^{2}}\left(\mathbf{e}_{i} \cdot \mathbf{u}\right)+\frac{9}{2 c^{4}}\left(\mathbf{e}_{i} \cdot \mathbf{u}\right)^{2}-\frac{3}{2 c^{2}}|\mathbf{u}|^{2}\right]\right\},
$$

where $\omega_{i}$ is given by $\omega_{0}=1 / 3, \omega_{1-6}=1 / 18$, and $\omega_{7-18}=$ $1 / 36$, and $\phi_{i}^{k}$ is employed to incorporate the pressure of fluid $k$; i.e., $p_{k}=\rho_{k}\left(c_{s}^{k}\right)^{2}$. The following perturbation operator $\left(\Omega_{i}^{k}\right)^{(2)}$ was adopted [31]:

$$
\left(\Omega_{i}^{k}\right)^{(2)}=A|\mathbf{C}|\left[\frac{\left(\mathbf{e}_{i} \cdot \mathbf{C}\right)^{2}}{|\mathbf{C}|^{2}}-\frac{5}{9}\right],
$$

where $\mathbf{C}$ is the color gradient and the free parameter $A$ is proportional to the surface tension.

Another three-dimensional color-gradient LB model can be found in the study of Liu et al. [27]. They employed the recoloring algorithm proposed by Latva-Kokko and Rothman [25], which can solve the lattice pinning problem and reduce the spurious velocities. In the algorithm of Latva-Kokko and Rothman, the streaming process of the LB equation is written as

$$
f_{i}^{k}\left(\mathbf{x}+\mathbf{e}_{i} \delta_{t}, t+\delta_{t}\right)=f_{i}^{k,+}(\mathbf{x}, t),
$$

where $f_{i}^{k,+}(\mathbf{x}, t)$ is obtained by the following recoloring steps [25,32]:

$$
\begin{aligned}
& f_{i}^{R,+}=\frac{\rho_{R}}{\rho} f_{i}^{*}+\beta \frac{\rho_{R} \rho_{B}}{\rho^{2}} \cos \left(\varphi_{i}\right) \sum_{k} f_{i}^{k, \mathrm{eq}}\left(\rho_{k}, \alpha_{k}, \mathbf{u}=0\right), \\
& f_{i}^{B,+}=\frac{\rho_{B}}{\rho} f_{i}^{*}-\beta \frac{\rho_{R} \rho_{B}}{\rho^{2}} \cos \left(\varphi_{i}\right) \sum_{k} f_{i}^{k, \mathrm{eq}}\left(\rho_{k}, \alpha_{k}, \mathbf{u}=0\right),
\end{aligned}
$$

where $\beta$ is a free parameter controlling the interface thickness and $f_{i}^{*}$ is the postperturbation value of the total distribution function, i.e., $f_{i}^{*}=\sum_{k} f_{i}^{*, k}$, in which $f_{i}^{*, k}$ is given by

$$
f_{i}^{*, k}(\mathbf{x}, t)=f_{i}^{k}(\mathbf{x}, t)+\left(\Omega_{i}^{k}\right)^{(1)}(\mathbf{x}, t)+\left(\Omega_{i}^{k}\right)^{(2)}(\mathbf{x}, t) .
$$

The parameter $\varphi_{i}$ in Eqs. (9) and (10) is the angle between the color gradient $\nabla \rho^{N}$ and the lattice direction $\mathbf{e}_{i}$, which gives $\cos \left(\varphi_{i}\right)=\left(\mathbf{e}_{i} \cdot \nabla \rho^{N}\right) /\left(\left|\mathbf{e}_{i}\right|\left|\nabla \rho^{N}\right|\right)$, where $\rho^{N}$ is defined as

$$
\rho^{N}=\frac{\left(\rho_{R} / \rho_{R}^{\text {in }}-\rho_{B} / \rho_{B}^{\text {in }}\right)}{\left(\rho_{R} / \rho_{R}^{\text {in }}+\rho_{B} / \rho_{B}^{\text {in }}\right)}
$$

in which $\rho_{R}^{\text {in }}$ and $\rho_{B}^{\text {in }}$ are the initial densities of the red and blue fluids, respectively. In numerical implementation, the color gradient $\nabla \rho^{N}$ is calculated by

$$
\nabla \rho^{N} \approx \frac{3}{c^{2} \delta_{t}} \sum_{i} \omega_{i} \rho^{N}\left(\mathbf{x}+\mathbf{e}_{i} \delta_{t}\right) \mathbf{e}_{i} .
$$

The work of Liu et al. [27] extended the perturbation operator of Reis and Phillips [26] to three-dimensional space,

$$
\left(\Omega_{i}^{k}\right)^{(2)}=\frac{A_{k}}{2}\left|\nabla \rho^{N}\right|\left[\omega_{i} \frac{\left(\mathbf{e}_{i} \cdot \nabla \rho^{N}\right)^{2}}{\left|\nabla \rho^{N}\right|^{2}}-B_{i}\right],
$$

where $B_{i}$ is given by $B_{0}=-1 / 3, B_{1-6}=1 / 18$, and $B_{7-18}=$ $1 / 36$. The above perturbation operator yields the following surface tension [27]:

$$
\sigma=\frac{2}{9}\left(A_{R}+A_{B}\right) \tau c^{4} \delta_{t} .
$$

The equilibrium distribution function is still defined by Eq. (6) but $\phi_{i}^{k}$ is given by [27]

$$
\phi_{i}^{k}= \begin{cases}\alpha_{k}, & i=0 \\ \left(1-\alpha_{k}\right) / 12, & i=1, \ldots, 6 \\ \left(1-\alpha_{k}\right) / 24, & i=7, \ldots, 18\end{cases}
$$

which corresponds to the pressure $p_{k}=\rho_{k}\left(c_{s}^{k}\right)^{2}=$ $0.5 \rho_{k} c^{2}\left(1-\alpha_{k}\right)$.

\section{Error terms}

The error terms in the momentum equation recovered from the three-dimensional color-gradient LB models have been identified by Huang et al. [28] through the Chapman-Enskog analysis and are given by

$$
\begin{aligned}
U_{\alpha}^{k}= & \partial_{\beta}\left\{\left(\tau-\frac{1}{2}\right)\left[\frac{1}{3} c^{2}-\left(c_{s}^{k}\right)^{2}\right]\right. \\
& \left.\times\left[u_{\beta} \partial_{\alpha} \rho_{k}+u_{\alpha} \partial_{\beta} \rho_{k}+\partial_{\gamma}\left(\rho_{k} u_{\gamma}\right) \delta_{\alpha \beta}\right]\right\},
\end{aligned}
$$

where the subscripts $\alpha, \beta$, and $\gamma$ denote the $x, y$, or $z$ coordinate and $\delta_{\alpha \beta}$ is the Kronecker delta. For two-phase flows with identical densities, $\phi_{i}^{k}=\omega_{i}$ is usually adopted and then $f_{i}^{k, \text { eq }}$ given by Eq. (6) reduces to the standard equilibrium distribution function in the LB method, which leads to $\left(c_{s}^{k}\right)^{2}=$ $c^{2} / 3$. Accordingly, the error terms disappear for two-phase flows with identical densities. However, for two-phase flows with different densities, the error terms in Eq. (17) will cause the Galilean invariance to be lost and may affect the numerical accuracy significantly since the density gradient cannot be neglected near the interface.

Recently, Saito et al. [33] constructed a three-dimensional 27-velocity (D3Q27) color-gradient LB model, in which an enhanced equilibrium distribution function devised by Leclaire et al. [34] is adopted

$$
\begin{aligned}
f_{i}^{k, \text { eq }}= & \rho_{k}\left[\phi_{i}^{k}+\omega_{i}\left[\frac{3}{c^{2}}\left(\mathbf{e}_{i} \cdot \mathbf{u}\right)+\frac{9}{2 c^{4}}\left(\mathbf{e}_{i} \cdot \mathbf{u}\right)^{2}-\frac{3}{2 c^{2}}|\mathbf{u}|^{2}\right]\right] \\
& +\Phi_{i}^{k},
\end{aligned}
$$

where $\Phi_{i}^{k}$ is an additional term given by

$$
\Phi_{i}^{k}= \begin{cases}-3 v\left(\mathbf{u} \cdot \nabla \rho_{k}\right) / c, & i=1, \\ +16 v\left(\boldsymbol{G}_{k}: \mathbf{e}_{i} \otimes \mathbf{e}_{i}\right) / c^{3}, & i=2,3, \ldots, 7, \\ +4 v\left(\boldsymbol{G}_{k}: \mathbf{e}_{i} \otimes \mathbf{e}_{i}\right) / c^{3}, & i=8,9, \ldots, 19, \\ +1 v\left(\boldsymbol{G}_{k}: \mathbf{e}_{i} \otimes \mathbf{e}_{i}\right) / c^{3}, & i=20,21, \ldots, 27,\end{cases}
$$

where $\boldsymbol{G}_{k}=\left[\mathbf{u} \otimes \nabla \rho_{k}+\left(\mathbf{u} \otimes \nabla \rho_{k}\right)^{\mathrm{T}}\right] / 48$. This additional term was originally employed by Che Sidik and Tanahashi [35] for a free-energy LB model and was extended to the color-gradient LB method by Leclaire et al. [34]. Nevertheless, it is noticed that both Che Sidik and Tanahashi [35] and Leclaire et al. [34] showed that there are still some error terms in the recovered macroscopic momentum equation, which can be found in Eqs. (29)-(33) of Ref. [34]. The main error terms are similar to the aforementioned error terms given by Eq. (17). 


\section{IMPROVED 3D COLOR-GRADIENT LB MODEL}

\section{A. Theoretical analysis}

In this section, the physical origin of the error terms in Eq. (17) is analyzed. Taking the second-order and third-order moments of the equilibrium distribution function given by Eq. (6), we can obtain

$$
\begin{gathered}
\sum_{i} e_{i \alpha} e_{i \beta} f_{i}^{k, \mathrm{eq}}=\rho_{k} u_{\alpha} u_{\beta}+\rho_{k}\left(c_{s}^{k}\right)^{2}, \\
\sum_{i} e_{i \alpha} e_{i \beta} e_{i \gamma} f_{i}^{k, \mathrm{eq}}=\rho_{k} \frac{c^{2}}{3}\left(u_{\alpha} \delta_{\beta \gamma}+u_{\beta} \delta_{\alpha \gamma}+u_{\gamma} \delta_{\alpha \beta}\right) .
\end{gathered}
$$

As seen in Eq. (20), the usual pressure $p=\rho_{k} c^{2} / 3$ has been replaced by $p_{k}=\rho_{k}\left(c_{s}^{k}\right)^{2}$. However, in the third-order moment given by Eq. (21), the pressure is still defined as $p=\rho_{k} c^{2} / 3$. Through the Chapman-Enskog analysis, it can be found that the error terms in Eq. (17) just arise from such an inconsistency. If $\rho_{k} c^{2} / 3$ in Eq. (21) can be replaced by $\rho_{k}\left(c_{s}^{k}\right)^{2}$, the error terms can be removed. However, the symmetry of standard lattices (such as the D2Q9, D3Q19, and D3Q27 lattices) is insufficient to completely support the replacement of $\rho_{k} c^{2} / 3$ in Eq. (21) with $\rho_{k}\left(c_{s}^{k}\right)^{2}$.

Fortunately, the off-diagonal elements of the third-order moment of the equilibrium distribution function can satisfy the required relationship by adding a high-order term to the equilibrium distribution function, as shown in Ref. [30] for recovering $p=\rho R T$ in a compressible LB model on standard lattices. Following the study of Li et al. [30], the improved equilibrium distribution function is defined as

$$
\begin{aligned}
f_{i}^{k, \text { eq }}= & \rho_{k}\left(\phi_{i}^{k}+\omega_{i}\left\{\frac{3}{c^{2}}\left(\mathbf{e}_{i} \cdot \mathbf{u}\right)+\frac{9}{2 c^{4}}\left(\mathbf{e}_{i} \cdot \mathbf{u}\right)^{2}-\frac{3}{2 c^{2}}|\mathbf{u}|^{2}\right.\right. \\
& \left.\left.+\frac{3\left(\mathbf{e}_{i} \cdot \mathbf{u}\right)}{2 c^{2}}\left[\frac{3\left(c_{s}^{k}\right)^{2}}{c^{2}}-1\right]\left(\frac{3\left|\mathbf{e}_{i}\right|^{2}}{c^{2}}-5\right)\right\}\right),
\end{aligned}
$$

which yields

$$
\begin{aligned}
& \sum_{i} e_{i \alpha} e_{i \beta} e_{i \gamma} f_{i}^{k, \text { eq }} \\
& \quad= \begin{cases}\rho_{k}\left(c^{2} / 3\right)\left(u_{\alpha} \delta_{\beta \gamma}+u_{\beta} \delta_{\alpha \gamma}+u_{\gamma} \delta_{\alpha \beta}\right), & \text { if } \alpha=\beta=\gamma \\
\rho_{k}\left(c_{s}^{k}\right)^{2}\left(u_{\alpha} \delta_{\beta \gamma}+u_{\beta} \delta_{\alpha \gamma}+u_{\gamma} \delta_{\alpha \beta}\right), & \text { others }\end{cases}
\end{aligned}
$$

For the off-diagonal elements of the third-order moment of $f_{i}^{k \text {, eq }}$, it can be seen that $\rho_{k} c^{2} / 3$ has been replaced by $\rho_{k}\left(c_{s}^{k}\right)^{2}$. Nevertheless, the diagonal elements $(\alpha=\beta=\gamma)$ still deviate from the required relationship owing to the low symmetry of standard lattices.

To remove the error terms caused by the diagonal elements of the third-order moment of $f_{i}^{k, \text { eq }}$, a correction term can be added to the single-phase collision operator,

$$
\begin{aligned}
\left(\Omega_{i}^{k}\right)^{(1)}= & -\frac{1}{\tau}\left[f_{i}^{k}(\mathbf{x}, t)-f_{i}^{k, \text { eq }}(\mathbf{x}, t)\right] \\
& +\delta_{t}\left(1-\frac{1}{2 \tau}\right) G_{i}^{k}(\mathbf{x}, t),
\end{aligned}
$$

where $G_{i}^{k}$ is the correction term and the coefficient $(1-0.5 / \tau)$ in front of $G_{i}^{k}$ is responsible for eliminating the discrete effect of the correction term [36]. The zerothand first-order moments of the correction term satisfy the following relationships:

$$
\sum_{i} G_{i}^{k}=0, \quad \sum_{i} \mathbf{e}_{i} G_{i}^{k}=0 .
$$

The constraints on the second-order moment of the correction term can be derived through the Chapman-Enskog analysis, which can be implemented by introducing the following multiscale expansions [37]:

$$
\begin{gathered}
f_{i}^{k}=f_{i}^{k,(0)}+\varepsilon f_{i}^{k,(1)}+\varepsilon^{2} f_{i}^{k,(2)}+\cdots, \\
\partial_{t}=\varepsilon \partial_{t_{1}}+\varepsilon^{2} \partial_{t_{2}}, \quad \nabla=\varepsilon \nabla_{1}, \quad G_{i}^{k}=\varepsilon G_{1 i}^{k},
\end{gathered}
$$

where $\varepsilon$ is the expansion parameter.

As pointed out by Grunau et al. [24], a color-gradient immiscible fluid model contains three fluid regions: homogeneous red and blue regions and a thin region near the interface where the two fluids mix. The recoloring step, which is subject to the constraints of mass conservation for the two fluids and momentum conservation for the mixture, occurs only in the mixed region. Accordingly, the recoloring step is usually not considered in the Chapman-Enskog analysis for deriving the hydrodynamic equations of the homogeneous regions, as can be seen in the studies of Grunau et al. [24], Reis and Phillips [26], and Liu et al. [27]. Meanwhile, the perturbation operator $\left(\Omega_{i}^{k}\right)^{(2)}$ only affects the surface tension term and has been well demonstrated in Refs. [26,27]. Hence, in the present study, the Chapman-Enskog analysis is performed for Eq. (1) with $\Omega_{i}^{k}(\mathbf{x}, t)$ being treated as $\Omega_{i}^{k}=\left(\Omega_{i}^{k}\right)^{(1)}$.

Taking the Taylor-series expansion of the left-hand side of Eq. (1) and using the multiscale expansions given by Eqs. (26) and (27), we can rewrite the LB equation in the consecutive orders of $\varepsilon$ as follows:

$$
\begin{gathered}
\mathrm{O}\left(\varepsilon^{0}\right): f_{i}^{k,(0)}=f_{i}^{k, \mathrm{eq}} \\
\mathrm{O}\left(\varepsilon^{1}\right):\left(\partial_{t_{1}}+\mathbf{e}_{i} \cdot \nabla_{1}\right) f_{i}^{k,(0)} \\
=-\frac{1}{\tau \delta_{t}} f_{i}^{k,(1)}+\left(1-\frac{1}{2 \tau}\right) G_{1 i}^{k}, \\
\mathrm{O}\left(\varepsilon^{2}\right): \partial_{t_{2}} f_{i}^{k,(0)}+\left(\partial_{t_{1}}+\mathbf{e}_{i} \cdot \nabla_{1}\right) f_{i}^{k,(1)} \\
+\frac{\delta_{t}}{2}\left(\partial_{t_{1}}+\mathbf{e}_{i} \cdot \nabla_{1}\right)^{2} f_{i}^{k,(0)}=-\frac{1}{\tau \delta_{t}} f_{i}^{k,(2)} .
\end{gathered}
$$

With the help of Eq. (29), Eq. (30) can be rewritten as

$$
\begin{aligned}
\mathrm{O}\left(\varepsilon^{2}\right): \partial_{t_{2}} f_{i}^{k,(0)}+\left(\partial_{t_{1}}+\mathbf{e}_{i} \cdot \nabla_{1}\right)\left(1-\frac{1}{2 \tau}\right) f_{i}^{k,(1)} \\
\quad+\frac{\delta_{t}}{2}\left(\partial_{t_{1}}+\mathbf{e}_{i} \cdot \nabla_{1}\right)\left(1-\frac{1}{2 \tau}\right) G_{1 i}^{k}=-\frac{1}{\tau \delta_{t}} f_{i}^{k,(2)} .
\end{aligned}
$$

Taking the summations of Eqs. (29) and (31) and using $\sum_{i} f_{i}^{k,(n)}=\sum_{i} \mathbf{e}_{i} f_{i}^{k,(n)}=0(n=1,2, \ldots)$ as well as Eq. (25), we can obtain

$$
\begin{gathered}
\partial_{t_{1}} \rho_{k}+\nabla_{1} \cdot\left(\rho_{k} \mathbf{u}\right)=0, \\
\partial_{t_{2}} \rho_{k}=0 .
\end{gathered}
$$


The continuity equation can be obtained by combining Eq. (32) with Eq. (33). Similarly, the first-order moments of Eqs. (29) and (31) yield, respectively,

$$
\begin{gathered}
\partial_{t_{1}}\left(\rho_{k} \mathbf{u}\right)+\nabla_{1} \cdot\left(\rho_{k} \mathbf{u u}\right)=-\nabla_{1} p_{k}, \\
\partial_{t_{2}}\left(\rho_{k} \mathbf{u}\right)+\nabla_{1} \cdot\left[\left(1-\frac{1}{2 \tau}\right) \sum_{i} \mathbf{e}_{i} \mathbf{e}_{i} f_{i}^{k,(1)}\right] \\
+\frac{\delta_{t}}{2} \nabla_{1} \cdot\left[\left(1-\frac{1}{2 \tau}\right) \sum_{i} \mathbf{e}_{i} \mathbf{e}_{i} G_{1 i}^{k}\right]=0,
\end{gathered}
$$

where $p_{k}=\rho_{k}\left(c_{s}^{k}\right)^{2}=0.5 \rho_{k} c^{2}\left(1-\alpha_{k}\right)$ with $\phi_{i}^{k}$ being given by Eq. (16). Meanwhile, Eq. (29) gives

$$
\begin{aligned}
\sum_{i} \mathbf{e}_{i} \mathbf{e}_{i} f_{i}^{k,(1)}= & -\tau \delta_{t}\left[\partial_{t_{1}}\left(\sum_{i} \mathbf{e}_{i} \mathbf{e}_{i} f_{i}^{k,(0)}\right)\right. \\
& +\nabla_{1} \cdot\left(\sum_{i} \mathbf{e}_{i} \mathbf{e}_{i} \mathbf{e}_{i} f_{i}^{k,(0)}\right) \\
& \left.-\left(1-\frac{1}{2 \tau}\right) \sum_{i} \mathbf{e}_{i} \mathbf{e}_{i} G_{1 i}^{k}\right] .
\end{aligned}
$$

Substituting Eq. (36) into Eq. (35) leads to

$$
\begin{gathered}
\partial_{t_{2}}\left(\rho_{k} \mathbf{u}\right)-\delta_{t} \nabla_{1} \cdot\left\{( \tau - 0 . 5 ) \left[\partial_{t_{1}}\left(\sum_{i} \mathbf{e}_{i} \mathbf{e}_{i} f_{i}^{k,(0)}\right)\right.\right. \\
\left.\left.+\nabla_{1} \cdot\left(\sum_{i} \mathbf{e}_{i} \mathbf{e}_{i} \mathbf{e}_{i} f_{i}^{k,(0)}\right)-\sum_{i} \mathbf{e}_{i} \mathbf{e}_{i} G_{1 i}^{k}\right]\right\} .
\end{gathered}
$$

Using the improved equilibrium distribution function given by Eq. (22), the off-diagonal elements of the third-order moment $\sum_{i} \mathbf{e}_{i} \mathbf{e}_{i} \mathbf{e}_{i} f_{i}^{k,(0)}$ can satisfy the required relationship, as shown in Eq. (23) (note that $f_{i}^{k,(0)}=f_{i}^{k, \text { eq }}$ ). Hence we can obtain the following constraints on the correction term $G_{i}^{k}$ :

$$
\sum_{i} e_{i x} e_{i y} G_{i}^{k}=\sum_{i} e_{i x} e_{i z} G_{i}^{k}=\sum_{i} e_{i y} e_{i z} G_{i}^{k}=0 .
$$

However, the diagonal elements of the third-order moment $\sum_{i} \mathbf{e}_{i} \mathbf{e}_{i} \mathbf{e}_{i} f_{i}^{k,(0)}$ deviate from the required relationship. To remove the related error terms, the correction term $G_{i}^{k}$ should satisfy

$$
\begin{aligned}
\sum_{i} e_{i x}^{2} G_{i}^{k} & =\partial_{x}\left\{\rho_{k} u_{x}\left[c^{2}-3\left(c_{s}^{k}\right)^{2}\right]\right\}, \\
\sum_{i} e_{i y}^{2} G_{i}^{k} & =\partial_{y}\left\{\rho_{k} u_{y}\left[c^{2}-3\left(c_{s}^{k}\right)^{2}\right]\right\}, \\
\sum_{i} e_{i z}^{2} G_{i}^{k} & =\partial_{z}\left\{\rho_{k} u_{z}\left[c^{2}-3\left(c_{s}^{k}\right)^{2}\right]\right\} .
\end{aligned}
$$

With these constraints, the error terms caused by the diagonal elements of the third-order moment of the equilibrium distribution function can be removed, and then the following equation can be derived from Eq. (37) by substituting Eqs. (20) and (23) as well as the above constraints into
Eq. (37):

$$
\partial_{t_{2}}\left(\rho_{k} \mathbf{u}\right)=\delta_{t} \nabla_{1} \cdot\left\{(\tau-0.5) p_{k}\left[\nabla_{1} \mathbf{u}+\left(\nabla_{1} \mathbf{u}\right)^{\mathrm{T}}\right]\right\} .
$$

Combining Eq. (42) with Eq. (34) using (27), the following macroscopic momentum equation can be obtained:

$\partial_{t}\left(\rho_{k} \mathbf{u}\right)+\nabla \cdot\left(\rho_{k} \mathbf{u u}\right)=-\nabla p_{k}+\nabla \cdot\left[\rho_{k} v_{k}\left(\nabla \mathbf{u}+(\nabla \mathbf{u})^{\mathrm{T}}\right)\right]$,

where the kinematic viscosity $v_{k}$ is given by $v_{k}=$ $(\tau-0.5) \delta_{t}\left(c_{s}^{k}\right)^{2}$.

To sum up, the improved equilibrium distribution function given by Eq. (22) and the correction term in Eq. (24) constitute the improvements for removing the error terms in Eq. (17). The form of the correction term can be determined by the aforementioned constraints. Particularly, since the constraints are given in the form of the moments of $G_{i}^{k}$, the correction term can be readily obtained in the moment space, namely, $\mathbf{C}^{k}=\mathbf{M G}^{k}$, where $\mathbf{C}^{k}$ is the correction term in the moment space and $\mathbf{M}$ is the transformation matrix of an MRT collision operator. Considering such a feature of the MRT collision operator and its better numerical stability over the BGK collision operator, in what follows we shall construct the improved three-dimensional color-gradient LB model based on the MRT collision operator.

\section{B. Improved model based on the MRT collision operator}

Using the MRT collision operator [38-41], the singlephase collision operator with a correction term can be written as follows:

$$
\begin{aligned}
\left(\Omega_{i}^{k}\right)^{(1)}(\mathbf{x}, t)= & -\Lambda_{i j}\left[f_{j}^{k}(\mathbf{x}, t)-f_{j}^{k, \mathrm{eq}}(\mathbf{x}, t)\right] \\
& +\delta_{t}\left(\delta_{i j}-\frac{1}{2} \Lambda_{i j}\right) G_{j}(\mathbf{x}, t),
\end{aligned}
$$

where $\delta_{i j}$ is the Kronecker delta and $\Lambda_{i j}=\left(\mathbf{M}^{-1} \mathbf{S M}\right)_{i j}$, in which $\mathbf{M}$ is the transformation matrix and $\mathbf{S}$ is a diagonal matrix for the relaxation times. Obviously, when $\Lambda_{i j}=\delta_{i j} / \tau$ (i.e., the BGK collision operator), Eq. (44) reduces to Eq. (24). Through the transformation matrix, the right-hand side of Eq. (44) can be implemented in the moment space:

$$
\overline{\mathbf{m}}^{k}=-\mathbf{S}\left(\mathbf{m}^{k}-\mathbf{m}^{k, \mathrm{eq}}\right)+\delta_{t}\left(\mathbf{I}-\frac{\mathbf{S}}{2}\right) \mathbf{C}^{k}
$$

where $\mathbf{I}$ is the unit matrix, $\mathbf{m}^{k}=\mathbf{M f}^{k}$ is the related moments, $\mathbf{m}^{k \text {,eq }}$ is the equilibria in the moment space, and $\mathbf{C}^{k}=\mathbf{M G}^{k}$ is the correction term in the moment space. Hence Eq. (44) can be rewritten as

$$
\left(\Omega_{i}^{k}\right)^{(1)}=\left(\mathbf{M}^{-1}\right)_{i j}\left(\overline{\mathbf{m}}^{k}\right)_{j} .
$$

The recoloring steps for the red and blue fluids are still given by Eqs. (9) and (10), respectively.

In the present work, the improved color-gradient MRT-LB model is constructed using the D3Q19 lattice and the transformation matrix $\mathbf{M}$ is based on the following basis vectors $[42,43]$ :

(i) the zeroth-order and first-order vectors, which are the vectors related to the conserved moments:

$$
M_{0, i}=1, \quad M_{1, i}=e_{i x}, \quad M_{2, i}=e_{i y}, \quad M_{3, i}=e_{i z},
$$


(ii) the second-order vectors related to the viscous effect at the Navier-Stokes level:

$$
\begin{aligned}
& M_{4, i}=\left|\mathbf{e}_{i}\right|^{2}, \quad M_{5, i}=3 e_{i x}^{2}-\left|\mathbf{e}_{i}\right|^{2}, \quad M_{6, i}=e_{i y}^{2}-e_{i z}^{2}, \\
& M_{7, i}=e_{i x} e_{i y}, \quad M_{8, i}=e_{i x} e_{i z}, \quad M_{9, i}=e_{i y} e_{i z},
\end{aligned}
$$

(iii) the third-order vectors:

$$
\begin{array}{lll}
M_{10, i}=e_{i x}^{2} e_{i y}, & M_{11, i}=e_{i x} e_{i y}^{2}, & M_{12, i}=e_{i x}^{2} e_{i z}, \\
M_{13, i}=e_{i x} e_{i z}^{2}, & M_{14, i}=e_{i y}^{2} e_{i z}, & M_{15, i}=e_{i y} e_{i z}^{2},
\end{array}
$$

(iv) the fourth-order vectors:

$$
M_{16, i}=e_{i x}^{2} e_{i y}^{2}, \quad M_{17, i}=e_{i x}^{2} e_{i z}^{2}, \quad M_{18, i}=e_{i y}^{2} e_{i z}^{2} .
$$

The first ten basis vectors are related to the macroscopic density, momentum, and the viscous stress tensor, whereas the other vectors are related to high-order moments that do not affect the Navier-Stokes level hydrodynamics. The detailed form of the transformation matrix $\mathbf{M}$ is given in the Appendix and its inverse matrix can be found in Ref. [43]. The relaxation matrix $\mathbf{S}$ in Eq. (45) is defined as [42,43]

$$
\begin{aligned}
\mathbf{S}= & \operatorname{diag}\left(1,1,1,1, \tau_{e}^{-1}, \tau_{v}^{-1}, \tau_{v}^{-1}, \tau_{v}^{-1}, \tau_{v}^{-1}, \tau_{v}^{-1}, \tau_{q}^{-1},\right. \\
& \left.\tau_{q}^{-1}, \tau_{q}^{-1}, \tau_{q}^{-1}, \tau_{q}^{-1}, \tau_{q}^{-1}, \tau_{\pi}^{-1}, \tau_{\pi}^{-1}, \tau_{\pi}^{-1}\right),
\end{aligned}
$$

where the relaxation times $\tau_{v}$ and $\tau_{e}$ determine the shear and bulk viscosities, respectively, while $\tau_{q}$ and $\tau_{\pi}$ are related to nonhydrodynamic moments.

The equilibria in the moment space can be obtained by $\mathbf{m}^{k, \text { eq }}=\mathbf{M} \mathbf{f}^{k, \text { eq }}$ (see the Appendix for details), in which $\mathbf{f}^{k, \text { eq }}=\left(f_{0}^{k \text {, eq }}, f_{1}^{k \text {, eq }}, \ldots, f_{18}^{k, \text { eq }}\right)^{\mathbf{T}}$ is given by Eq. (22), i.e., the improved equilibrium density distribution function. Similarly, the correction term in the moment space can be derived from $\mathbf{C}^{k}=\mathbf{M G}^{k}$. According to the constraints on $G_{i}^{k}$ [see Eqs. (25) and (38)-(41)], the following correction term can be obtained in the moment space:

$$
\mathbf{C}^{k}=\left[\begin{array}{c}
0 \\
0 \\
0 \\
0 \\
Q_{x}+Q_{y}+Q_{z} \\
2 Q_{x}-Q_{y}-Q_{z} \\
Q_{y}-Q_{z} \\
0 \\
0 \\
0 \\
0 \\
0 \\
0 \\
0 \\
0 \\
0 \\
0 \\
0 \\
0
\end{array}\right],
$$

where $Q_{x}, Q_{y}$, and $Q_{z}$ are given by [see also Eqs. (39)-(41)], respectively,

$$
Q_{x}=\partial_{x}\left\{\rho_{k} u_{x}\left[c^{2}-3\left(c_{s}^{k}\right)^{2}\right]\right\},
$$

$$
\begin{aligned}
Q_{y} & =\partial_{y}\left\{\rho_{k} u_{y}\left[c^{2}-3\left(c_{s}^{k}\right)^{2}\right]\right\}, \\
Q_{z} & =\partial_{z}\left\{\rho_{k} u_{z}\left[c^{2}-3\left(c_{s}^{k}\right)^{2}\right]\right\},
\end{aligned}
$$

where $\left(c_{s}^{k}\right)^{2}=0.5 c^{2}\left(1-\alpha_{k}\right)$. The high-order moments of $G_{i}^{k}$ have been set to zero in deriving Eq. (52). In numerical implementation, the second-order isotropic difference scheme is applied to the spatial gradients in Eqs. (53)-(55), i.e.,

$$
Q_{\alpha} \approx \frac{3}{c^{2} \delta_{t}} \sum_{i} \omega_{i} \bar{Q}_{\alpha}\left(\mathbf{x}+\mathbf{e}_{i} \delta_{t}\right) e_{i \alpha},
$$

where $\alpha$ denotes the $x, y$, or $z$ coordinate and $\bar{Q}_{\alpha}=$ $\rho_{k} u_{\alpha}\left[c^{2}-3\left(c_{s}^{k}\right)^{2}\right]$. It can be found that such a calculation is the same as the calculation of the color gradient $\nabla \rho^{N}$ in Eq. (13).

The Chapman-Enskog analysis can also be applied to the MRT collision operator, which is similar to that of the BGK collision operator. Readers are referred to Refs. [43-45] about the Chapman-Enskog analysis of the three-dimensional MRT-LB method. It can be found that, using the equilibria $\mathbf{m}^{k, \text { eq }}$ in the Appendix and the correction term $\mathbf{C}^{k}$ given by Eq. (52), the following macroscopic momentum equation can be derived in the low Mach number limit:

$$
\begin{aligned}
\partial_{t}\left(\rho_{k} \mathbf{u}\right)+\nabla \cdot\left(\rho_{k} \mathbf{u} \mathbf{u}\right)= & -\nabla p_{k}+\nabla \cdot\left\{\mu_{k}\left[\nabla \mathbf{u}+(\nabla \mathbf{u})^{\mathrm{T}}\right]\right. \\
& \left.-\frac{2}{3} \mu_{k}(\nabla \cdot \mathbf{u}) \mathbf{I}+\mu_{k}^{b}(\boldsymbol{\nabla} \cdot \mathbf{u}) \mathbf{I}\right\},
\end{aligned}
$$

where the dynamic shear viscosity $\mu_{k}$ and the bulk viscosity $\mu_{k}^{b}$ are given by, respectively,

$$
\mu_{k}=\rho_{k}\left(c_{s}^{k}\right)^{2}\left(\tau_{v}-\frac{1}{2}\right) \delta_{t}, \quad \mu_{k}^{b}=\frac{2}{3} \rho_{k}\left(c_{s}^{k}\right)^{2}\left(\tau_{e}-\frac{1}{2}\right) \delta_{t} .
$$

The kinematic viscosity $v_{k}$ is given by $v_{k}=\mu_{k} / \rho_{k}$. When $\tau_{e}=$ $\tau_{v}$, Eq. (57) reduces to Eq. (43).

To ensure the smoothness of the relaxation time $\tau_{v}$ (corresponding to $\tau$ in the BGK model) across the interface, $\tau_{v}$ is calculated as follows $[24,26]$ :

$$
\tau_{v}=\left\{\begin{array}{ll}
\tau_{v}^{R}, & \rho^{N}>\delta \\
g^{R}\left(\rho^{N}\right), & \delta \geqslant \rho^{N}>0 \\
g^{B}\left(\rho^{N}\right), & 0 \geqslant \rho^{N} \geqslant-\delta \\
\tau_{v}^{B}, & \rho^{N}<-\delta
\end{array},\right.
$$

where $\delta$ is a free parameter related to the interface thickness and is usually set as $\delta=0.98$ [14], and $g^{R}$ and $g^{B}$ are parabolic functions of $\rho^{N}$ [its definition is given by Eq. (12)], as shown in Refs. [24,26]. The relaxation times $\tau_{v}^{R}$ and $\tau_{v}^{B}$ in Eq. (59) are determined by the kinematic viscosities of the red and blue fluids; i.e., $v_{R}=\left(c_{s}^{R}\right)^{2}\left(\tau_{v}^{R}-0.5\right) \delta_{t}$ and $v_{B}=$ $\left(c_{s}^{B}\right)^{2}\left(\tau_{v}^{B}-0.5\right) \delta_{t}$, respectively.

The surface tension in Eq. (15) depends on the relaxation time. A simple treatment to make the surface tension independent of the relaxation time is to change the perturbation operator from Eq. (14) to $\left(\Omega_{i}^{k}\right)^{(2) \text {, new }}=\frac{1}{\tau}\left(\Omega_{i}^{k}\right)^{(2)}$, and then the surface tension is given by $\sigma=2\left(A_{R}+A_{B}\right) c^{4} \delta_{t} / 9$. Correspondingly, the perturbation operator within the framework of the MRT-LB method can be redefined as follows:

$$
\left(\Omega_{i}^{k}\right)^{(2), \text { new }}=\Lambda_{i j}\left(\Omega_{j}^{k}\right)^{(2)} .
$$


Similar to the single-phase collision operator in Eq. (44), the perturbation operator given by Eq. (60) can also be executed in the moment space.

\section{NUMERICAL RESULTS AND DISCUSSION}

In this section, numerical simulations are carried out to validate the improved three-dimensional color-gradient LB model. First, the test of a moving droplet in a uniform flow field is employed to verify the Galilean invariance of the improved model. Subsequently, the numerical accuracy of the improved model is demonstrated through simulating the layered two-phase flow and three-dimensional Rayleigh-Taylor instability. Finally, the capability of the improved model for simulating dynamic two-phase flows at a large density ratio is validated by the simulation of droplet impact on a solid wall.

\section{A. Moving droplet in a uniform flow field}

In the LB community [46,47], it has been reported that a circular droplet in a uniform flow field will become an elliptic one when employing a two-phase LB model with broken Galilean invariance. To verify the Galilean invariance of the proposed improved color-gradient LB model, the test of a moving circular droplet in a uniform flow field is considered. Our simulations are carried out in a domain divided into $N_{x} \times N_{y}=140 \times 140$ lattices. A circular droplet of radius $r_{0}=$ 30 (lattice unit) is placed at the center of the computational domain and brought to the equilibrium state at rest. Then the two parallel plates in the $y$ direction begin to move with a constant velocity $U=0.02$ at $t=0$. The Zou-He boundary scheme [48] is applied in the $y$ direction and the periodic boundary condition is employed in the $x$ and $z$ directions.

The initial densities of the red and blue fluids are taken as $\rho_{R}^{\text {in }}=3$ and $\rho_{B}^{\text {in }}=1$, respectively, with $\alpha_{R}$ and $\alpha_{B}$ being set to 0.9 and 0.7 , respectively, which satisfy $\rho_{R}^{\text {in }} / \rho_{B}^{\text {in }}=$ $\left(1-\alpha_{B}\right) /\left(1-\alpha_{R}\right)$ [29]. The parameters $A_{R}$ and $A_{B}$ for the surface tension are $A_{R}=A_{B}=0.01$ and the parameter $\beta$ in Eqs. (9) and (10) is chosen as $\beta=0.5$. The relaxation time $\tau_{v}$ is determined by Eq. (59) and the other relaxation times are set to 1.0. The dynamic viscosities are chosen as $\mu_{R}=\mu_{B}=$ 0.075 . Figure 1 shows the simulated snapshots of a moving circular droplet. For comparison, the numerical results of the color-gradient MRT-LB model without the improvements are also presented, which is the MRT version of the threedimensional color-gradient LB model of Liu et al. [27] and is hereinafter referred to as the original model. When the original model is employed, the shape of the droplet becomes elliptic, as shown in Fig. 1(a), which means that the lack of Galilean invariance leads to deformation of the droplet. On the contrary, from Fig. 1(b) we can see that the improved color-gradient model allows the droplet to retain its circular shape, demonstrating that the Galilean invariance is restored in the improved model.

\section{B. Layered two-phase flow in a channel}

In this subsection, the layered two-phase flow between two parallel plates is simulated to validate the numerical accuracy of the improved color-gradient LB model. As shown
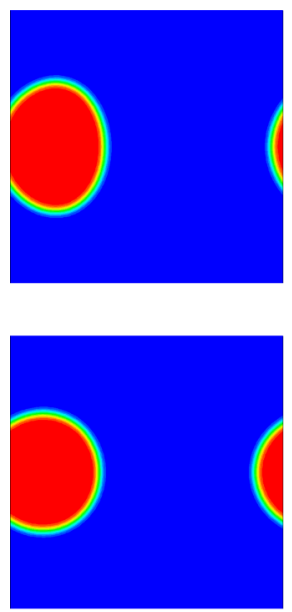

FIG. 1. Density contours of a moving droplet simulated by (a) the original model and (b) the improved model. From left to right: $t=60000 \delta_{t}, 80000 \delta_{t}$, and $95000 \delta_{t}$.

in Fig. 2, the channel height is $h=2 b$ in the $y$ direction with $y=0$ at the center of the channel. The red fluid is initially located in the central region $-a \leqslant y \leqslant a$, whereas the blue fluid is located in the regions $a<|y| \leqslant b$. The layered twophase flow is driven by a constant body force $(G, 0,0)$. By assuming a Poiseuille-type flow in the channel, we can obtain the following the analytical solution for the velocity profile [14]:

$$
u_{x}(y)=\left\{\begin{array}{ll}
A_{1} y^{2}+C_{1} & (0 \leqslant|y| \leqslant a) \\
A_{2} y^{2}+B_{2} y+C_{2} & (a \leqslant|y| \leqslant b)
\end{array},\right.
$$

where the coefficients are defined as

$$
\begin{aligned}
& A_{1}=-\frac{G}{2 \rho_{R} \nu_{R}}, \quad A_{2}=-\frac{G}{2 \rho_{B} v_{B}}, \quad B_{2}=2\left(A_{1} M-A_{2}\right) a, \\
& C_{1}=\left(A_{2}-A_{1}\right) a^{2}-B_{2}(b-a)-A_{2} b^{2}, \\
& C_{2}=-A_{2} b^{2}-B_{2} b,
\end{aligned}
$$

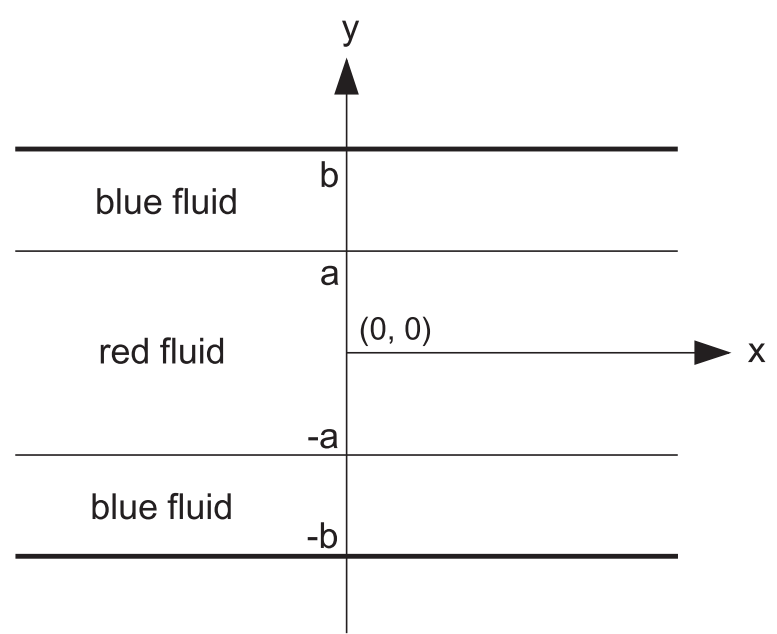

FIG. 2. Schematic of the layered two-phase flow between two parallel plates. 


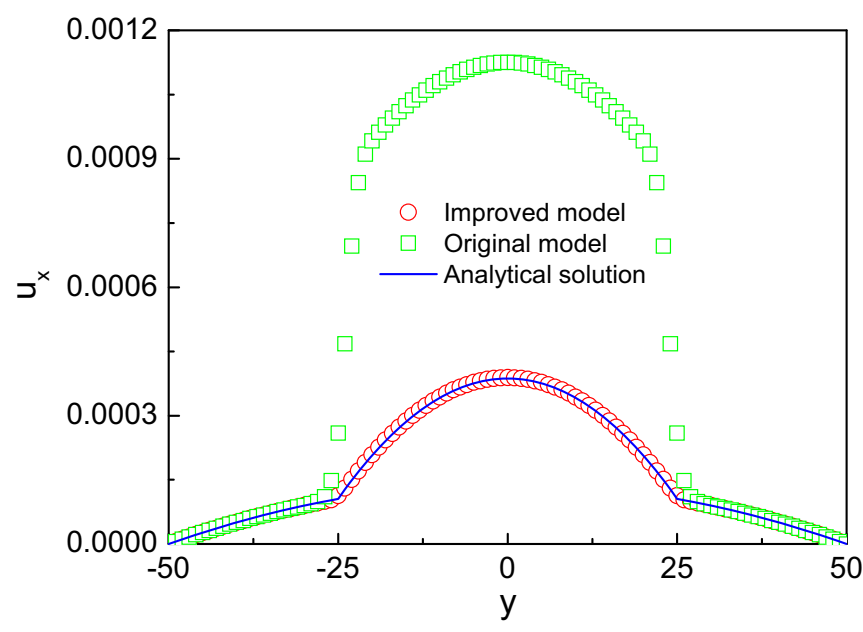

(a)

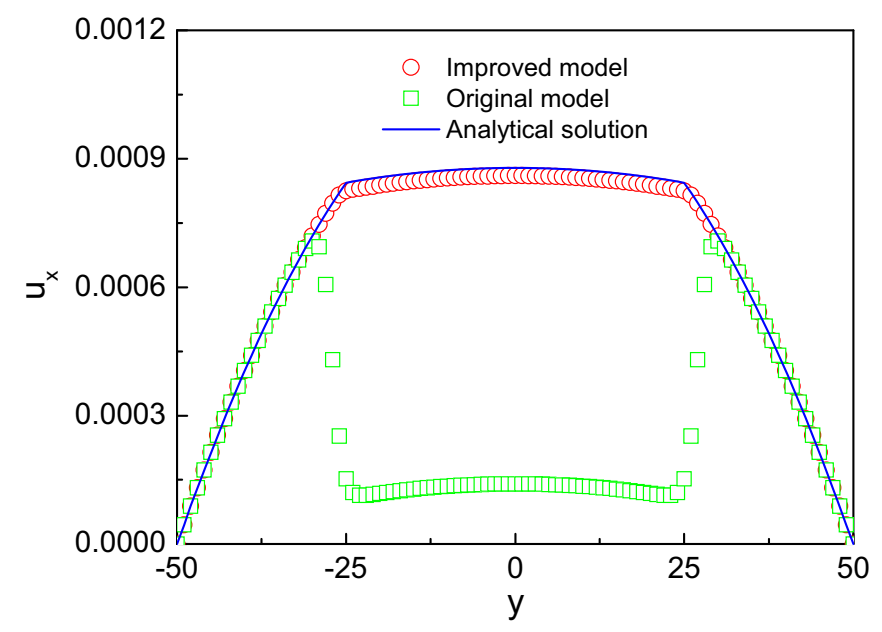

(b)

FIG. 3. Simulation of layered two-phase flow in a channel. Comparison of the velocity profiles obtained by the original and improved color-gradient LB models for (a) case A and (b) case B.

in which $M=\mu_{R} / \mu_{B}$ is the dynamic shear viscosity ratio [14].

In our simulations, the computational domain is divided into $N_{x} \times N_{y}=10 \times 100$ lattices with $a=25$ and $b=50$. The nonslip boundary condition [48] is applied to the two parallel plates, while the periodic boundary condition is employed in the $x$ and $z$ directions. Three cases are investigated:

Case A: $\rho_{R}^{\text {in }}=0.1, \quad \rho_{B}^{\text {in }}=0.8, \quad \alpha_{B}=0.9, \quad M=1 / 8 ;$

Case B: $\rho_{R}^{\text {in }}=0.8, \quad \rho_{B}^{\text {in }}=0.1, \quad \alpha_{B}=0.2, \quad M=8$;

Case C: $\rho_{R}^{\text {in }}=0.008, \quad \rho_{B}^{\text {in }}=8, \quad \alpha_{B}=0.9992, \quad M=1 / 40$.

The parameter $\alpha_{R}$ is determined via $\rho_{R}^{\text {in }} / \rho_{B}^{\text {in }}=\left(1-\alpha_{B}\right) /$ $\left(1-\alpha_{R}\right)$. The parameters $A_{R}$ and $A_{B}$ for the surface tension are chosen as $A_{R}=A_{B}=0.0001$. The constant body force in the $x$ direction is taken as $G=1.5 \times 10^{-8}$.

Figures 3 and 4 display the numerical results obtained by the original and improved color-gradient LB models for cases A-C. For comparison, the analytical solutions are also presented. From the figures we can see that the velocity profiles predicted by the improved model are in good agreement with the analytical solutions, whereas the numerical results obtained by the original model significantly deviate from the analytical ones. Particularly, it can be seen that the main numerical errors appear within the interval $y \in[-25,25]$. This is because the error terms in Eq. (17) cannot be neglected due to the abrupt change of the momentum across the interfaces around $y= \pm 25$. To quantify the numerical simulations, the relative error between the numerical results and the analytical solutions is evaluated, which is defined as $E_{u}=\sum_{y}\left|u_{x}(y)-u_{x}^{a}(y)\right| / \sum_{y}\left|u_{x}^{a}(y)\right|$, where $u_{x}^{a}(y)$ denotes the analytical solution. For the improved model, the relative errors of cases $\mathrm{A}-\mathrm{C}$ are $E_{u}=0.66 \%, 1.42 \%$, and $0.36 \%$, respectively, while the relative errors yielded by the original model for the three cases are $E_{u}=44.6 \%, 59.7 \%$, and $6904 \%$, respectively. Here it can be seen that the error of case $\mathrm{C}$ caused by the original model is much larger than those of cases A and
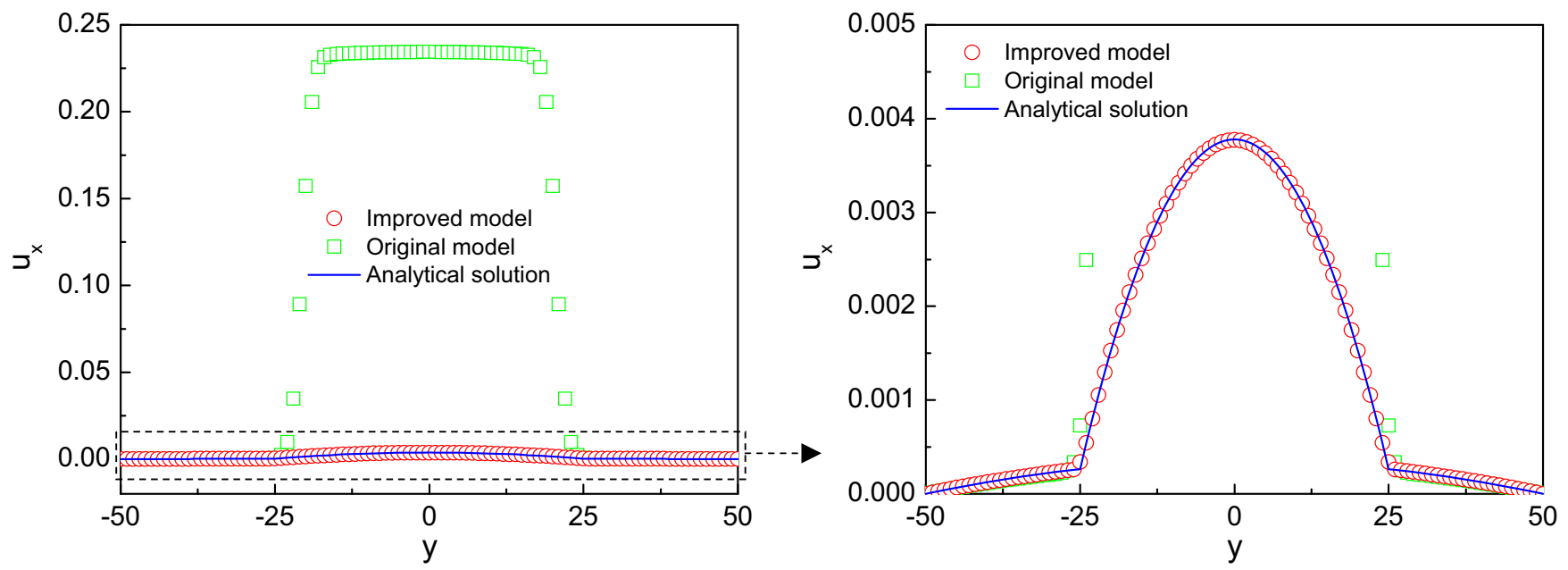

FIG. 4. Simulation of layered two-phase flow in a channel. Comparison of the velocity profiles obtained by the original and improved color-gradient LB models for case C. 


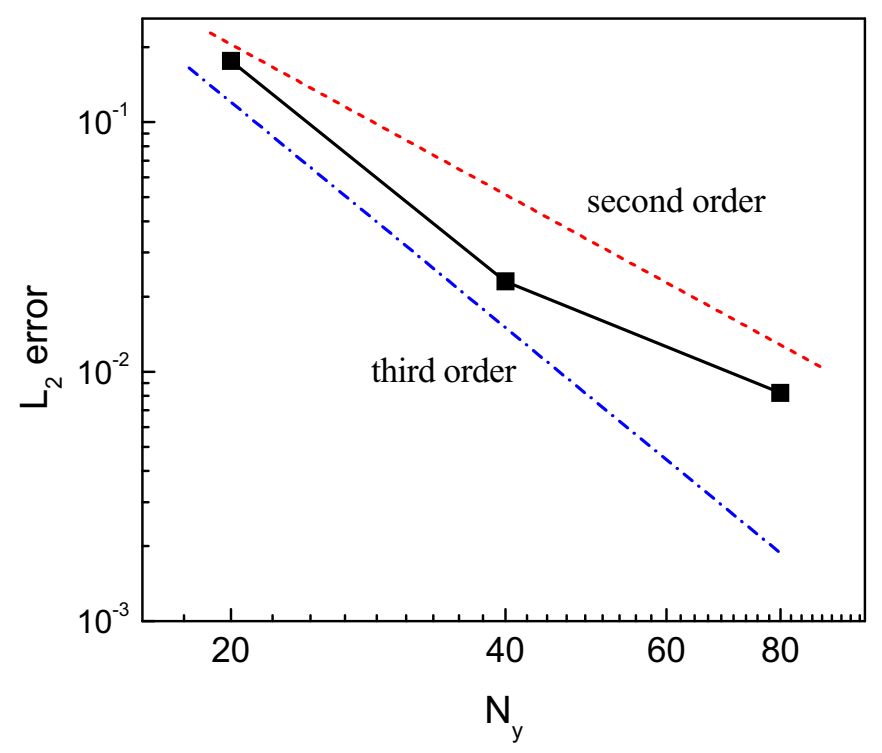

FIG. 5. Convergence order of the present color-gradient LB model.

$\mathrm{B}$, which is attributed to the fact that the ratio $\rho_{B}^{\mathrm{in}} / \rho_{R}^{\mathrm{in}}$ is very large in case $C$. Besides, it is observed that the results of case $\mathrm{C}$ obtained by the present model are also much better than those obtained by the model of Ba et al. [29] [see Fig. 3(e) in their paper]. The convergence order of the present model is examined. Figure 5 plots the relative $L_{2}$-norm errors of case $\mathrm{C}$ (the density ratio is 1000) against the grid size in the $y$ direction. As can be seen in the figure, the present model tends to be second-order accurate.

\section{Three-dimensional Rayleigh-Taylor instability}

The phenomenon of Rayleigh-Taylor instability is associated with the penetration of a heavy fluid into a light fluid and can be found in a wide range of scientific and environmental fields. This problem involves complex interfacial interactions and has been intensively studied because of its practical and scientific importance [49-51]. It consists of two layers of fluid at rest; a heavy fluid is on top of a light fluid. The heavy fluid accelerates into the light fluid under the action of the gravity. In the present study, the test of three-dimensional RayleighTaylor instability is employed to investigate the capability of the improved model for modeling two-phase flows with complex interfacial interactions. The computational domain is a rectangular box of $\Omega=[0, L] \times[0, L] \times[0,4 L]$. The nonslip boundary condition is applied to the upper and lower solid walls, while the periodic boundary condition is employed at the four vertical boundaries.

In our simulations, the red (heavy) fluid is placed above the blue (light) fluid and the Atwood number At $=$ $\left(\rho_{R}^{\text {in }}-\rho_{B}^{\text {in }}\right) /\left(\rho_{R}^{\text {in }}+\rho_{B}^{\text {in }}\right)$ is set to 0.5 for the sake of comparing our numerical results with those reported in the literature [51]. The Reynolds number is defined as $\operatorname{Re}=L \sqrt{L g} / \nu$, where $g$ is the gravitational acceleration and $v$ is the kinematic viscosity. In this problem, the kinematic viscosities of the two fluids are identical. The characteristic velocity of the system is taken as $U=\sqrt{L g}=0.04, L$ is chosen as $L=125$ (lattice unit), and the Reynolds number is set to $\operatorname{Re}=1024$. The investi- gated Rayleigh-Taylor instability develops from the following single-mode initial perturbation:

$$
\frac{h(x, y)}{L}=0.05\left[\cos \left(\frac{2 \pi x}{L}\right)+\cos \left(\frac{2 \pi y}{L}\right)\right],
$$

where $h$ is the height of the fluid interface.
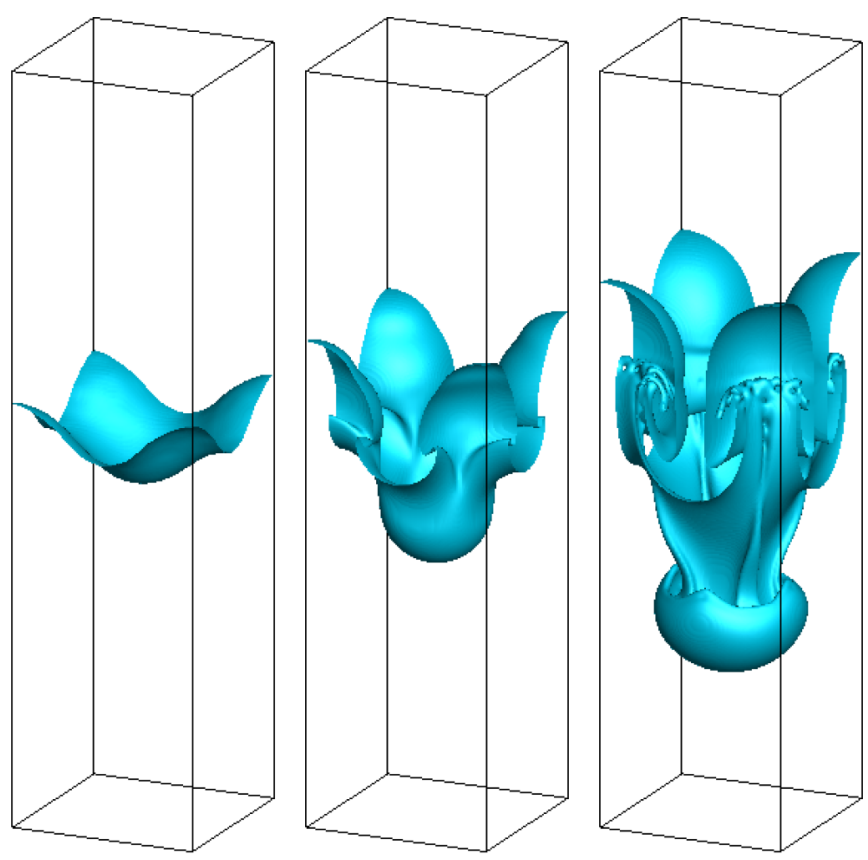

(a)
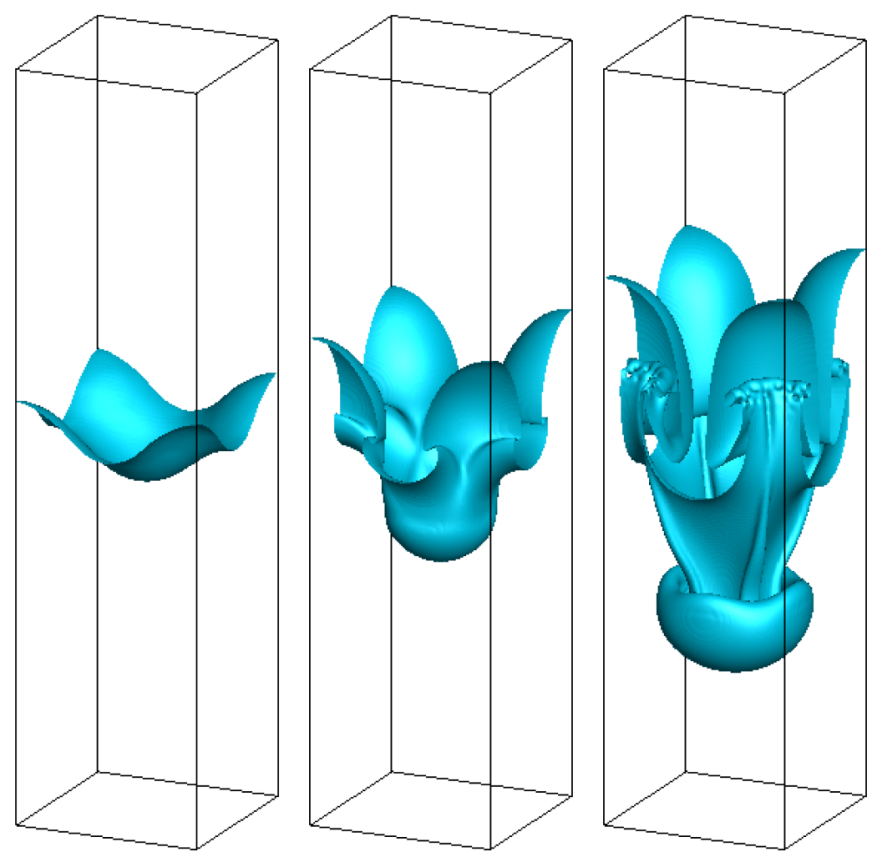

(b)

FIG. 6. Simulation of three-dimensional Rayleigh-Taylor instability. Snapshots of the fluid interface obtained by (a) the original model and (b) the improved model at $t^{*}=1,2$, and 3 (from left to right). 


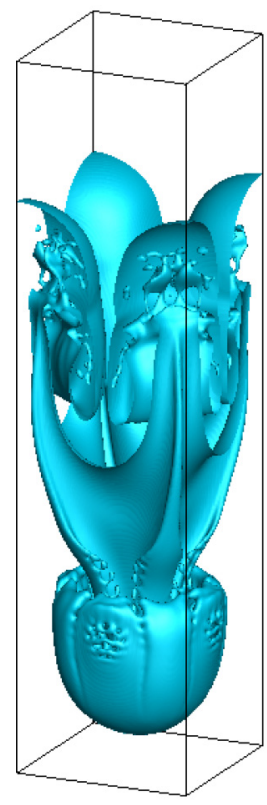

(a)

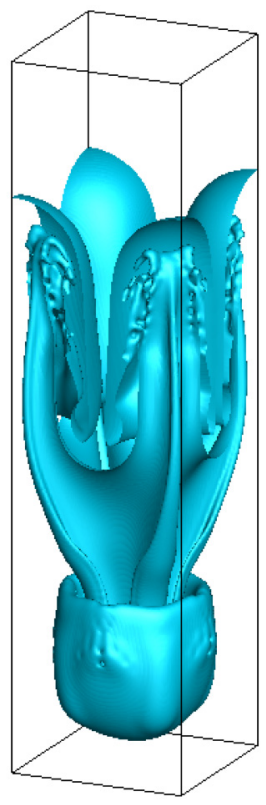

(b)

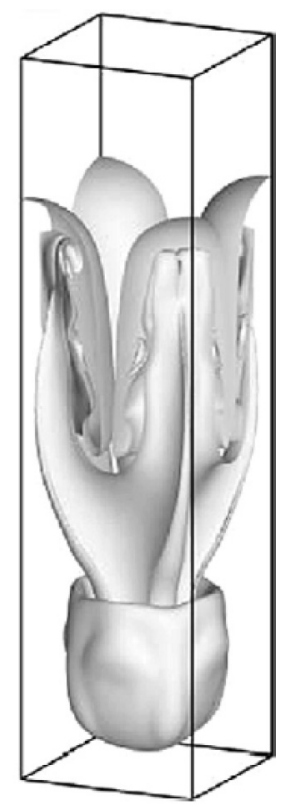

(c)
FIG. 7. Comparison of the fluid interface at $t^{*}=4$ obtained by (a) the original color-gradient model, (b) the improved color-gradient model, and (c) a multiphase flux solver in Ref. [51].

Figure 6 shows the evolution of the fluid interface simulated by the original and improved color-gradient models at $t^{*}=1,2$, and 3 , where the time $t^{*}$ is nondimensional and is normalized by the reference time $t_{\text {ref }}=\sqrt{L / g}$. From the numerical results of both models we can see that the heavy and light fluids penetrate into each other as time increases. Specifically, at $t^{*}=1$ it can be seen that a spike is formed in the middle due to the downward movement of the heavy fluid and bubbles are formed on the sides because of the rising of light fluid. At the early stage, the numerical results of the two models show the same interface shapes. Subsequently, the first roll-up of the heavy fluid appears in the neighborhood of the saddle points, as can be seen at $t^{*}=2$, and we can find that the shapes of the bubbles become a little different for the original and improved models. Later, at $t^{*}=3$ the second roll-up takes place at the edge of the spike. At this time, the shapes of the spike obtained by the original and improved models are quite different, which is attributed to the fact that the downward velocity of the spike gradually increases and the error term in Eq. (17) accordingly becomes non-negligible.

As time goes by, significant differences can be observed between the results of the improved model and those of the original model, as shown in Fig. 7, which shows a comparison of the fluid interface at $t^{*}=4$ obtained by the original model, the improved model, and a multiphase flux solver in Ref. [51]. At this stage, two extra layers of the heavy fluid are folded upward as a result of the stretch of the two roll-ups: One forms a skirt around the spike and the other forms a girdle inside the bubble. Particularly, it can be seen that the shapes of the spike and girdle predicted by the original model obviously deviate from those obtained by a multiphase flux solver [51], while the shapes simulated by the improved model are in good

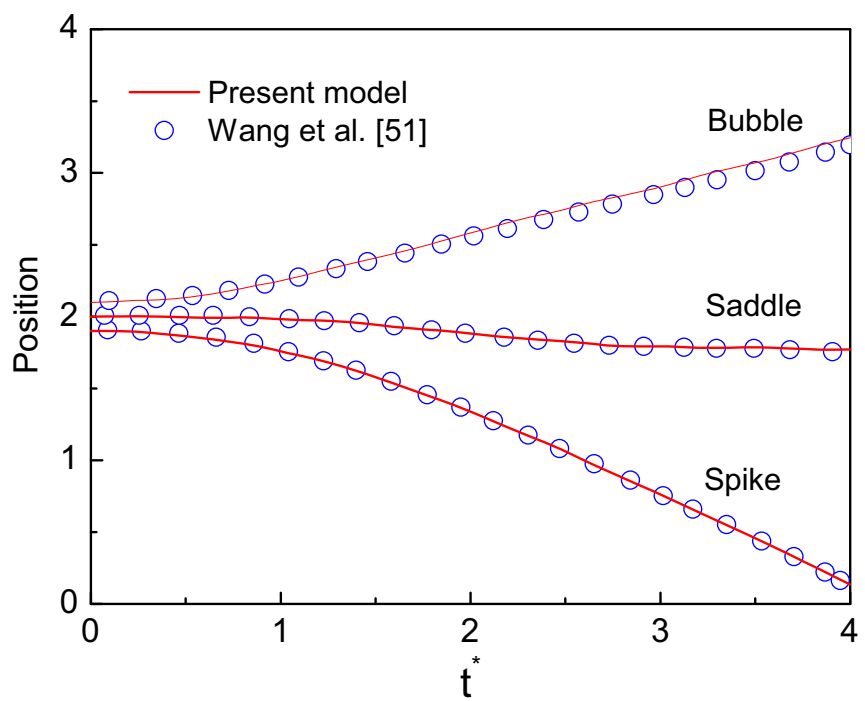

FIG. 8. The positions of the bubble front, the spike tip, and the saddle point versus time. The Atwood number is 0.5 and the Reynolds number is 1024 .

agreement with those reported in Ref. [51]. Figure 8 depicts the evolution of the interface positions of the bubble front, the spike tip, and the saddle point. As shown in the figure, the interface positions predicted by our improved model agree well with the results of Wang et al. [51]. Table I gives a quantitative comparison of the position of the spike tip between the original and improved models. From the table we can see that, when $t^{*}<3.2$, there are just slight differences between the results of the original model and those of the improved model. However, the differences gradually increase and considerable deviations can be observed at $t^{*}=4$. This is because the error term given by Eq. (17) is dependent on the velocity. Accordingly, the errors caused by the error term are negligible when the velocity is relatively small and cannot be neglected as the velocity increases considerably.

\section{Droplet impact on a solid surface}

Finally, the impingement of a droplet on a flat surface is simulated to validate the capability of the proposed colorgradient MRT-LB model for simulating dynamic two-phase flows at a relatively large density ratio. Impingement of droplets on a solid surface is a very important phenomenon in many engineering applications, such as ink-jet printing and spray cooling. The dynamics of droplet impact on solid surfaces is usually governed by the following two nondimensional parameters:

$$
\operatorname{Re}=\frac{\rho_{R} U_{0} D_{0}}{\mu_{R}}, \quad \mathrm{We}=\frac{\rho_{R} U_{0}^{2} D_{0}}{\sigma},
$$

TABLE I. Comparison of the position of spike tip between the original and improved models.

\begin{tabular}{lccccccc}
\hline \hline$t^{*}$ & 1.6 & 2.4 & 2.8 & 3.2 & 3.44 & 3.76 & 4.0 \\
\hline Original & 1.5331 & 1.1266 & 0.8886 & 0.6506 & 0.5073 & 0.3124 & 0.1631 \\
Improved & 1.5315 & 1.1204 & 0.8793 & 0.6407 & 0.4946 & 0.2921 & 0.1367 \\
\hline
\end{tabular}


where Re and We are the Reynolds number and the Weber number, respectively. In Eq. (64), $U_{0}$ is the impact speed of the droplet and $D_{0}$ is the initial diameter of the droplet.

The computational domain is divided into $N_{x} \times N_{y} \times N_{z}=$ $300 \times 300 \times 150$ lattices. The nonslip boundary condition is employed at the solid surface and the periodic condition is applied in the $x$ and $y$ directions. Initially, a spherical droplet of diameter $D_{0}=100$ (lattice unit) is placed on the center of the bottom flat surface. The initial velocity of the droplet is given by $\mathbf{u}_{0}=\left(u_{x}, u_{y}, u_{z}\right)=\left(0,0,-U_{0}\right)$, in which $U_{0}=$ 0.006. The initial densities of the red and blue fluids are given by $\rho_{R}^{\text {in }}=8$ and $\rho_{B}^{\text {in }}=0.08$, respectively, with $\alpha_{B}=0.2$. The equilibrium contact angle of the flat surface is taken as $\theta \approx 90^{\circ}$ and the parameters $A_{R}$ and $A_{B}$ for the surface tension are chosen as $A_{R}=A_{B}=0.00225$, which leads to the surface tension $\sigma \approx 0.001$. Correspondingly, the Weber number is $\mathrm{We} \approx 28.8$.

In our simulations, the Reynolds number varies from $\mathrm{Re}=$ 75 to 1000 . Figure 8 displays some snapshots of the droplet impingement process at $\mathrm{Re}=1000$. As shown in the figure, immediately after the impingement, the shape of the droplet resembles a truncated sphere [Fig. 9(a)]. Later, a lamella is formed as the liquid moves radially outward [Figs. 9(b) and 9(c)]. The lamella continues to grow radially [Fig. 8(d)] until the maximum spreading diameter is reached and the spreading process ends, during which the kinetic energy is transformed into the surface energy by increasing the area of the droplet [51]. After reaching the maximum spreading diameter, the lamella begins to retract because of the surface tension, as can be seen in Figs. 9(e) and 9(f). These observations agree well with those reported in Refs. [52-55].

To quantify the numerical results, the maximum spreading factor $D_{\max } / D_{0}$ obtained by the proposed color-gradient LB model is compared with the data reported in the literature.

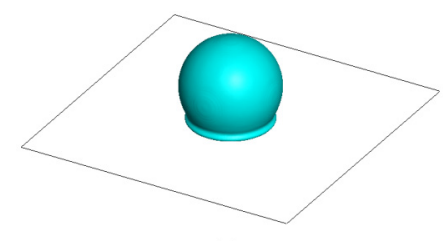

(a)

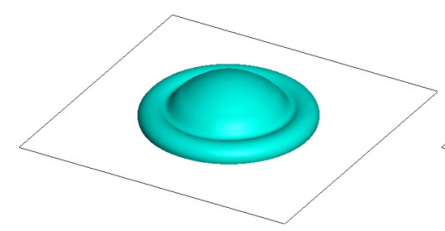

(c)

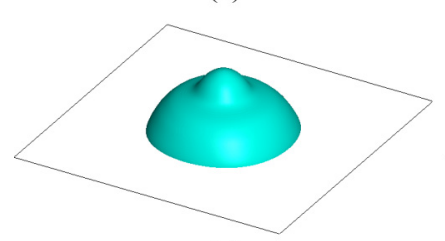

(e)

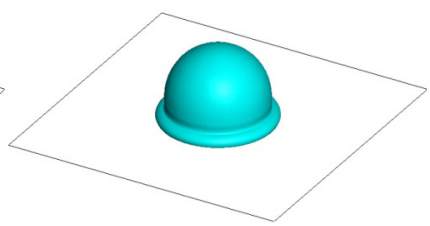

(b)

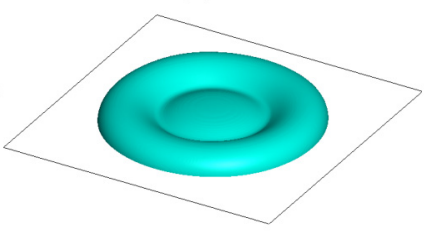

(d)

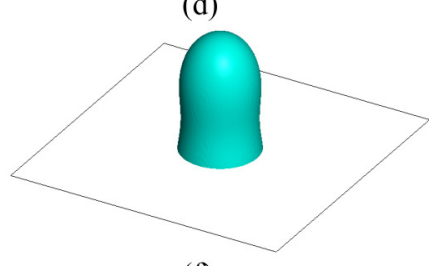

(f)
FIG. 9. Snapshots of droplet impact on a flat surface at $\mathrm{Re}=$ 1000 with $\rho_{R} / \rho_{B}=100$. (a) $t=2000 \delta_{t}$, (b) $t=4000 \delta_{t}$, (c) $t=$ $10000 \delta_{t}$, (d) $t=30000 \delta_{t}$, (e) $t=60000 \delta_{t}$, and (f) $t=90000 \delta_{t}$.

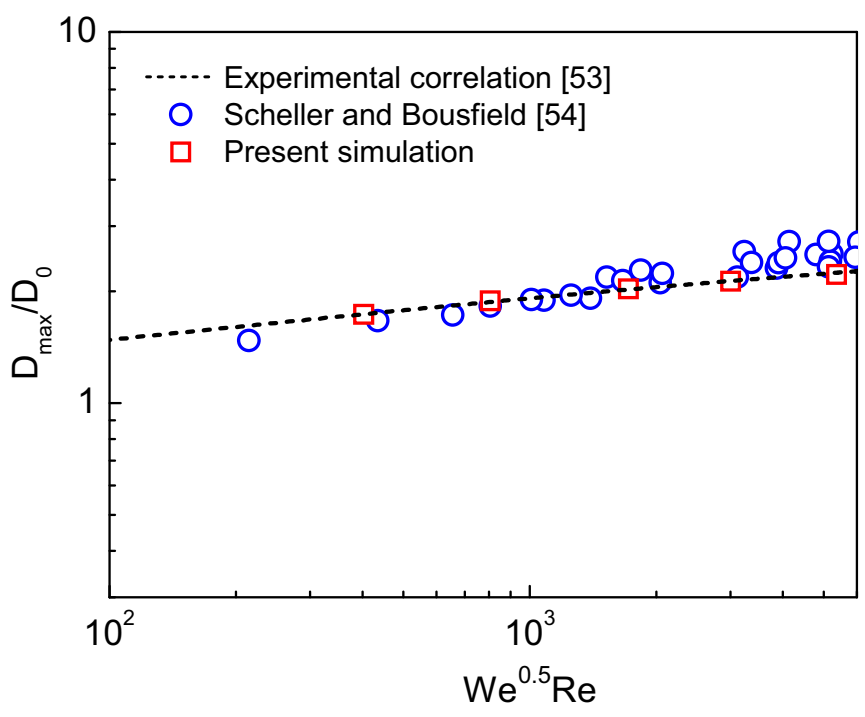

FIG. 10. Comparison of the maximum spreading factor between the present numerical results, the experimental correlation in Ref. [53], and the experimental data in Ref. [54].

In Ref. [53], Asai et al. established the following correlation formula for the maximum spreading factor based on their experimental data:

$$
D_{\max } / D_{0}=1+0.48 \mathrm{We} \mathrm{e}^{0.5} \exp \left(-1.48 \mathrm{We} \mathrm{e}^{0.22} \mathrm{Re}^{-0.21}\right) .
$$

In addition, Scheller and Bousfield [54] also proposed a correlation formula by plotting their experimental data versus $\mathrm{OhRe}^{2}=\sqrt{\mathrm{We}} \mathrm{Re}$, in which $\mathrm{Oh}=\sqrt{\mathrm{We}} / \mathrm{Re}=\mu_{R} / \sqrt{\rho_{R} \sigma D_{0}}$ is the Ohnesorge number. Figure 10 shows a comparison of the maximum spreading factor between the experimental correlation formula of Asai et al. [53], the experimental data of Scheller and Bousfield [54], and the numerical results predicted by the proposed color-gradient LB model. From the figure it can be seen that our numerical results are in good agreement with the experimental correlation and data reported in the previous studies, demonstrating that the improved colorgradient LB model is capable of simulating dynamic twophase flows at a relatively large density ratio.

\section{CONCLUSIONS}

The previous three-dimensional color-gradient LB models usually suffer from the lack of Galilean invariance and considerable numerical errors because of the error terms in the recovered macroscopic equations. In this paper we have theoretically analyzed the physical origin of the error terms in the previous models. Based on the theoretical analysis, we have proposed an improved three-dimensional color-gradient LB model for simulating immiscible two-phase flows. Specifically, a high-order term is added to the equilibrium distribution function, through which the off-diagonal elements of the third-order moment of the equilibrium distribution function can satisfy the required relationship for recovering the correct Navier-Stokes equations. Meanwhile, the deviations of the diagonal elements are corrected via a correction term in the LB equation. Compared with the previous models, the present 
model eliminates the error terms and therefore improves the numerical accuracy and enhances the Galilean invariance.

To validate the proposed color-gradient LB model, numerical simulations have been performed. The test of a moving droplet in a uniform flow field has been employed to verify the Galilean invariance of the improved model. It has been shown that the shape of the droplet becomes elliptic when the original model is used, while the improved model allows the droplet to retain its circular shape. $\mathrm{Nu}$ merical simulations have also been carried out for the layered two-phase flow and three-dimensional Rayleigh-Taylor instability, which show that the numerical accuracy of the improved model has been significantly improved in compar- ison with the original model. Furthermore, the capability of the improved color-gradient LB model for simulating dynamic two-phase flows at a relatively large density ratio has been demonstrated by simulating droplet impact on a solid surface.

\section{ACKNOWLEDGMENTS}

This work was supported by the National Natural Science Foundation of China (Grant No. 51822606) and the UK Consortium on Mesoscale Engineering Sciences (UKCOMES) under the UK Engineering and Physical Sciences Research Council Grant No. EP/R029598/1.

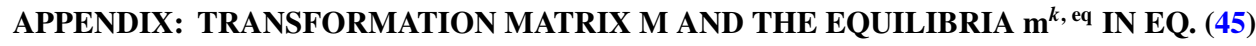

$$
\mathbf{M}=\left[\begin{array}{ccccccccccccccccccc}
1 & 1 & 1 & 1 & 1 & 1 & 1 & 1 & 1 & 1 & 1 & 1 & 1 & 1 & 1 & 1 & 1 & 1 & 1 \\
0 & 1 & -1 & 0 & 0 & 0 & 0 & 1 & -1 & 1 & -1 & 1 & -1 & 1 & -1 & 0 & 0 & 0 & 0 \\
0 & 0 & 0 & 1 & -1 & 0 & 0 & 1 & -1 & -1 & 1 & 0 & 0 & 0 & 0 & 1 & -1 & 1 & -1 \\
0 & 0 & 0 & 0 & 0 & 1 & -1 & 0 & 0 & 0 & 0 & 1 & -1 & -1 & 1 & 1 & -1 & -1 & 1 \\
0 & 1 & 1 & 1 & 1 & 1 & 1 & 2 & 2 & 2 & 2 & 2 & 2 & 2 & 2 & 2 & 2 & 2 & 2 \\
0 & 2 & 2 & -1 & -1 & -1 & -1 & 1 & 1 & 1 & 1 & 1 & 1 & 1 & 1 & -2 & -2 & -2 & -2 \\
0 & 0 & 0 & 1 & 1 & -1 & -1 & 1 & 1 & 1 & 1 & -1 & -1 & -1 & -1 & 0 & 0 & 0 & 0 \\
0 & 0 & 0 & 0 & 0 & 0 & 0 & 1 & 1 & -1 & -1 & 0 & 0 & 0 & 0 & 0 & 0 & 0 & 0 \\
0 & 0 & 0 & 0 & 0 & 0 & 0 & 0 & 0 & 0 & 0 & 1 & 1 & -1 & -1 & 0 & 0 & 0 & 0 \\
0 & 0 & 0 & 0 & 0 & 0 & 0 & 0 & 0 & 0 & 0 & 0 & 0 & 0 & 0 & 1 & 1 & -1 & -1 \\
0 & 0 & 0 & 0 & 0 & 0 & 0 & 1 & -1 & -1 & 1 & 0 & 0 & 0 & 0 & 0 & 0 & 0 & 0 \\
0 & 0 & 0 & 0 & 0 & 0 & 0 & 1 & -1 & 1 & -1 & 0 & 0 & 0 & 0 & 0 & 0 & 0 & 0 \\
0 & 0 & 0 & 0 & 0 & 0 & 0 & 0 & 0 & 0 & 0 & 1 & -1 & -1 & 1 & 0 & 0 & 0 & 0 \\
0 & 0 & 0 & 0 & 0 & 0 & 0 & 0 & 0 & 0 & 0 & 1 & -1 & 1 & -1 & 0 & 0 & 0 & 0 \\
0 & 0 & 0 & 0 & 0 & 0 & 0 & 0 & 0 & 0 & 0 & 0 & 0 & 0 & 0 & 1 & -1 & -1 & 1 \\
0 & 0 & 0 & 0 & 0 & 0 & 0 & 0 & 0 & 0 & 0 & 0 & 0 & 0 & 0 & 1 & -1 & 1 & -1 \\
0 & 0 & 0 & 0 & 0 & 0 & 0 & 1 & 1 & 1 & 1 & 0 & 0 & 0 & 0 & 0 & 0 & 0 & 0 \\
0 & 0 & 0 & 0 & 0 & 0 & 0 & 0 & 0 & 0 & 0 & 1 & 1 & 1 & 1 & 0 & 0 & 0 & 0 \\
0 & 0 & 0 & 0 & 0 & 0 & 0 & 0 & 0 & 0 & 0 & 0 & 0 & 0 & 0 & 1 & 1 & 1 & 1
\end{array}\right] .
$$

$$
\mathbf{m}^{k, \mathrm{eq}}=\left[\begin{array}{c}
\rho_{k} \\
\rho_{k} u_{x} \\
\rho_{k} u_{y} \\
\rho_{k} u_{z} \\
3 p_{k}+\rho_{k}|\mathbf{u}|^{2} \\
\rho_{k}\left(2 u_{x}^{2}-u_{y}^{2}-u_{z}^{2}\right) \\
\rho_{k}\left(u_{y}^{2}-u_{z}^{2}\right) \\
\rho_{k} u_{x} u_{y} \\
\rho_{k} u_{x} u_{z} \\
\rho_{k} u_{y} u_{z} \\
p_{k} u_{y} \\
p_{k} u_{x} \\
p_{k} u_{z} \\
p_{k} u_{x} \\
p_{k} u_{z} \\
p_{k} u_{y} \\
{\left[\rho_{k}\left(1-\alpha_{k}-|\mathbf{u}|^{2}\right)+2 \rho_{k} c^{2}\left(u_{x}^{2}+u_{y}^{2}\right)\right] / 6} \\
{\left[\rho_{k}\left(1-\alpha_{k}-|\mathbf{u}|^{2}\right)+2 \rho_{k} c^{2}\left(u_{x}^{2}+u_{z}^{2}\right)\right] / 6} \\
{\left[\rho_{k}\left(1-\alpha_{k}-|\mathbf{u}|^{2}\right)+2 \rho_{k} c^{2}\left(u_{y}^{2}+u_{z}^{2}\right)\right] / 6}
\end{array}\right],
$$

where $p_{k}=\rho_{k}\left(c_{s}^{k}\right)^{2}=0.5 \rho_{k} c^{2}\left(1-\alpha_{k}\right)$. 
[1] G. R. McNamara and G. Zanetti, Phys. Rev. Lett. 61, 2332 (1988).

[2] Y. H. Qian, D. d'Humières, and P. Lallemand, Europhys. Lett. 17, 479 (1992).

[3] R. Benzi, S. Succi, and M. Vergassola, Phys. Rep. 222, 145 (1992).

[4] F. J. Higuera, S. Succi, and R. Benzi, Europhys. Lett. 9, 345 (1989).

[5] S. Chen and G. D. Doolen, Annu. Rev. Fluid Mech. 30, 329 (1998).

[6] C. K. Aidun and J. R. Clausen, Annu. Rev. Fluid Mech. 42, 439 (2010).

[7] Q. Li, K. H. Luo, Q. J. Kang, Y. L. He, Q. Chen, and Q. Liu, Prog. Energy Combust. Sci. 52, 62 (2016).

[8] S. Succi, Europhys. Lett. 109, 50001 (2015).

[9] Z. Guo and C. Shu, Lattice Boltzmann Method and Its Applications in Engineering (World Scientific, Singapore, 2013).

[10] A. Xu, W. Shyy, and T. Zhao, Acta Mech. Sin. 33, 555 (2017).

[11] Y.-L. He, Q. Liu, Q. Li, and W.-Q. Tao, Int. J. Heat Mass Transfer 129, 160 (2019).

[12] U. Frisch, B. Hasslacher, and Y. Pomeau, Phys. Rev. Lett. 56, 1505 (1986).

[13] S. Succi, Eur. Phys. J. B 64, 471 (2008).

[14] H. Huang, M. Sukop, and X. Lu, Multiphase Lattice Boltzmann Methods: Theory and Application (John Wiley \& Sons, New York, 2015).

[15] A. K. Gunstensen, D. H. Rothman, S. Zaleski, and G. Zanetti, Phys. Rev. A 43, 4320 (1991).

[16] D. H. Rothman and J. M. Keller, J. Stat. Phys. 52, 1119 (1988).

[17] T. Krüger, H. Kusumaatmaja, A. Kuzmin, O. Shardt, G. Silva, and E. M. Viggen, The Lattice Boltzmann Method-Principles and Practice (Springer Nature, Berlin, 2017).

[18] X. Shan and H. Chen, Phys. Rev. E 47, 1815 (1993).

[19] X. Shan and H. Chen, Phys. Rev. E 49, 2941 (1994).

[20] M. R. Swift, W. R. Osborn, and J. M. Yeomans, Phys. Rev. Lett. 75, 830 (1995).

[21] M. R. Swift, E. Orlandini, W. R. Osborn, and J. M. Yeomans, Phys. Rev. E 54, 5041 (1996).

[22] D. Jacqmin, J. Comput. Phys. 155, 96 (1999).

[23] H. Liu, Q. Kang, C. R. Leonardi, S. Schmieschek, A. Narváez, B. D. Jones, J. R. Williams, A. J. Valocchi, and J. Harting, Comput. Geosci. 20, 777 (2016).

[24] D. Grunau, S. Chen, and K. Eggert, Phys. Fluids A 5, 2557 (1993).

[25] M. Latva-Kokko and D. H. Rothman, Phys. Rev. E 71, 056702 (2005).

[26] T. Reis and T. N. Phillips, J. Phys. A: Math. Theor. 40, 4033 (2007).

[27] H. Liu, A. J. Valocchi, and Q. Kang, Phys. Rev. E 85, 046309 (2012).
[28] H. Huang, J.-J. Huang, X.-Y. Lu, and M. C. Sukop, Int. J. Mod. Phys. C 24, 1350021 (2013).

[29] Y. Ba, H. Liu, Q. Li, Q. Kang, and J. Sun, Phys. Rev. E 94, 023310 (2016).

[30] Q. Li, K. H. Luo, Y. L. He, Y. J. Gao, and W. Q. Tao, Phys. Rev. E 85, 016710 (2012).

[31] J. Tölke, M. Krafczyk, M. Schulz, and E. Rank, Philos. Trans. 360, 535 (2002).

[32] S. Leclaire, M. Reggio, and J. Y. Trépanier, Appl. Math. Modell. 36, 2237 (2012).

[33] S. Saito, Y. Abe, and K. Koyama, Phys. Rev. E 96, 013317 (2017).

[34] S. Leclaire, N. Pellerin, M. Reggio, and J.-Y. Trépanier, Int. J. Multiphase Flow 57, 159 (2013).

[35] N. A. Che Sidik and T. Tanahashi, J. Mek. 24, 68 (2007).

[36] Z. Guo, C. Zheng, and B. Shi, Phys. Rev. E 65, 046308 (2002).

[37] J. M. Buick and C. A. Greated, Phys. Rev. E 61, 5307 (2000).

[38] P. Lallemand and L.-S. Luo, Phys. Rev. E 61, 6546 (2000).

[39] Q. Li, Y. L. He, G. H. Tang, and W. Q. Tao, Phys. Rev. E 81, 056707 (2010).

[40] L.-S. Luo, W. Liao, X. Chen, Y. Peng, and W. Zhang, Phys. Rev. E 83, 056710 (2011).

[41] Q. Li, K. H. Luo, and X. J. Li, Phys. Rev. E 87, 053301 (2013).

[42] Y. Yu, Z. X. Wen, Q. Li, P. Zhou, and H. J. Yan, Appl. Therm. Eng. 142, 846 (2018).

[43] Q. Li, D. H. Du, L. L. Fei, and K. H. Luo, Comput. Fluids 186, 128 (2019).

[44] K. N. Premnath and J. Abraham, J. Comput. Phys. 224, 539 (2007).

[45] A. Xu, T. S. Zhao, L. An, and L. Shi, Int. J. Heat Fluid Flow 56, 261 (2015).

[46] T. Inamuro, N. Konishi, and F. Ogino, Comput. Phys. Commun. 129, 32 (2000).

[47] A. N. Kalarakis, V. N. Burganos, and A. C. Payatakes, Phys. Rev. E 65, 056702 (2002).

[48] Q. Zou and X. He, Phys. Fluids 9, 1591 (1997).

[49] X. He, R. Zhang, S. Chen, and G. D. Doolen, Phys. Fluids 11, 1143 (1999).

[50] Q. Li, K. H. Luo, Y. J. Gao, and Y. L. He, Phys. Rev. E 85, 026704 (2012).

[51] Y. Wang, C. Shu, and L. M. Yang, J. Comput. Phys. 302, 41 (2015).

[52] D. Zhang, K. Papadikis, and S. Gu, Int. J. Multiphase Flow 64, 11 (2014).

[53] A. Asai, M. Shioya, S. Hirasawa, and T. Okazaki, J. Imaging Sci. Technol. 37, 205 (1993).

[54] B. L. Scheller and D. W. Bousfield, AIChE J. 41, 1357 (1995).

[55] A. Gupta and R. Kumar, Comput. Fluids 39, 1696 (2010). 